\title{
Limitantes físicos y bióticos de la regeneración arbórea en matorrales sucesionales de la Isla Grande de Chiloé, Chile
}

\author{
Physical and biotic constraints on tree regeneration in secondary shrublands of Chiloé \\ Island, Chile
}

MARÍA F. DÍAZ ${ }^{1,2, *} \&$ JUAN J. ARMESTO ${ }^{2,3}$

\begin{abstract}
${ }^{1}$ Laboratorio de Sistemática y Ecología Vegetal, Facultad de Ciencias, Universidad de Chile, Santiago, Chile ${ }^{2}$ Centro de Estudios Avanzados en Ecología y Biodiversidad, Pontificia Universidad Católica de Chile, Facultad de Ciencias Biológicas, Departamento de Ecología, Casilla 114-D, Santiago, Chile

${ }^{3}$ Instituto de Ecología \& Biodiversidad, Facultad de Ciencias, Universidad de Chile, Santiago, Chile

* e-mail para correspondencia: fdiazi@bio.puc.cl
\end{abstract}

\begin{abstract}
RESUMEN
Los matorrales sucesionales establecidos después de la corta y quema de bosques son frecuentes en Chiloé y territorios continentales vecinos. Estos sitios son dominados por arbustos de Baccharis patagonica, musgos en cojín del género Sphagnum, plantas palustres y helechos. En estos sitios hemos registrado escasa o nula colonización arbórea $\left(<0,3\right.$ plántulas $\left.\mathrm{m}^{-2}\right)$ comparado con 7,2 plántulas $\mathrm{m}^{-2}$ en bosques inmediatamente adyacentes. La colonización de árboles pioneros sombra-intolerantes podría estar limitada, entre otros factores, por acceso a luz de las plántulas bajo la densa cobertura de Baccharis, o por la presencia del musgo Sphagnum magellanicum, el cual genera sustratos orgánicos anegados, pobres en nutrientes, ácidos y aislantes del calor. Para evaluar estas hipótesis se realizaron ensayos de germinación de semillas de tres especies leñosas pioneras en gradientes experimentales de humedad del suelo, que simulaban las variaciones de humedad postperturbación en sitios dominados por matorral. Aunque la germinación fue baja o nula para Nothofagus y Drimys, no hubo efectos de las diferencias en humedad del suelo sobre la germinación, pero sí del tipo de sustrato. Embothrium coccineum presentó mayor germinación y supervivencia sobre sustrato de Sphagnum magellanicum. Se evaluó mediante trasplantes, la supervivencia de plántulas de dos especies de árboles pioneros (Embothrium coccineum y Drimys winteri) bajo y fuera de la cobertura de Baccharis y sobre el sustrato orgánico de Sphagnum magellanicum. Aunque la supervivencia de Embothrium y Drimys bajo Baccharis fue $<40 \%$, en uno de los sitios, no se observó diferencias entre tratamientos de cobertura ni sustrato. Los suelos anegados de las zonas de matorral serían limitantes para la regeneración de especies arbóreas pioneras debido a la falta de sustratos elevados. Sin embargo, la presencia de cojines de Sphagnum favorecería el establecimiento de Embothrium, especie que podría facilitar la restauración de estos hábitat.
\end{abstract}

Palabras clave: regeneración arbórea, Sphagnum magellanicum, Baccharis patagonica, supervivencia, germinación.

\begin{abstract}
Successional shrublands created by clearcutting and burning of forests are frequent in Chiloé Island and surrounding mainland in southern Chile. These areas are characterized by seasonally waterlogged soils, and vegetation dominated by sedges, ferns and shrubs, such as Baccharis patagonica, with thick carpets of Sphagnum moss occupying the spaces between shrubs. Tree regeneration in these sites was shown to be sparse or completely lacking $\left(<0.3\right.$ seedlings $\left.\mathrm{m}^{-2}\right)$ compared with 7.2 seedlings $\mathrm{m}^{-2}$ in adjacent forests. Colonization of shade-intolerant, pioneer trees may be reduced underneath Baccharis due to crown shading and on top of Sphagnum cushions, because of unfavorable conditions for tree establishment, including low $\mathrm{pH}$ and soil water saturation. We evaluated differences in germination rates among pioneer tree species grown in an experimental soil moisture gradient. Seed germination percentage was low for Drimys winteri and Nothofagus nitida unrelated to humidity conditions but differed between substrates (moss vs. soil). Embothrium had higher germination and survivorship when growing on Sphagnum cushions. We also monitored seedling survival of two pioneer tree species (Embothrium coccineum and Drimys winteri) transplanted directly under Baccharis shrubs, in open areas, or on moss patches. Even though survivorship of Embothrium and Drimys under Baccharis was $<40 \%$, in one of the study sites, there were no differences between treatments. Waterlogged soils would constraint tree regeneration on successional shrublands due to a lack of elevated
\end{abstract}


substrates. However, the presence of Sphagnum cushions favors Embothrium establishment, and thus may facilitate restoration of these sites.

Key words: tree regeneration, Sphagnum magellanicum, Baccharis patagonica, seedling survival, seed germination.

\section{INTRODUCCIÓN}

La teoría de sucesión explica el proceso de reemplazo de especies y el cambio en el desempeño de los individuos a través del tiempo en un mismo espacio. La primera fase de la sucesión secundaria, luego de una perturbación, es la invasión o regeneración de especies pioneras y el establecimiento de las plantas en el sitio perturbado (Pickett et al. 1987). La colonización de plántulas de árboles y arbustos en sitios perturbados es dependiente de varios procesos: la existencia de bancos de semillas, dispersión y depredación de semillas, emergencia y depredación de plántulas, y competencia entre plántulas.

Los procesos sucesionales son esencialmente demográficos y producen cambios en la estructura de la comunidad y funciones ecosistémicas (Pickett et al. 1987). Si las especies colonizadoras no permiten el establecimiento de especies sucesionales tardías por inhibición, la sucesión quedará detenida o retardada en sus etapas iniciales. Connell \& Slatyer (1977) propusieron tres modelos para explicar la influencia de plantas pioneras sobre la invasión y crecimiento de especies más tardías en la sucesión. Estos son: facilitación, inhibición y tolerancia. Especies sucesionales tempranas podrían tener efectos positivos (facilitación), negativos (inhibición) o no tener efecto (tolerancia) sobre el establecimiento de otras especies.

Diversos estudios en el hemisferio norte han investigado el proceso de colonización de árboles y arbustos en praderas abandonadas, donde las principales limitantes del proceso de sucesión son la disponibilidad de propágulos, la mortalidad de semillas y plántulas por efecto de herbívoros (De Steven 1991a, 1991b, Gill \& Marks 1991, Callaway 1992). En la Isla Grande de Chiloé, el cambio en el uso de suelo ha transformado el paisaje de bosques originalmente continuos a fragmentos de bosque rodeados de grandes extensiones de praderas y matorrales, que se observan estacionalmente anegados. El anegamiento se debe principalmente al pobre drenaje de los suelos orgánicos tipo ñadi sobre sustratos de origen fluvio-glacial (Aravena 1991, Veit \& Garleff 1996). En praderas abandonadas y estacionalmente húmedas, el arbusto Baccharis patagonica alcanza frecuencias superiores al 50 $\%$. Una vez que Baccharis se establece en el área, no se observa colonización arbórea, o esta es muy escasa (Papic 2000). La colonización de los árboles pioneros sombra-intolerantes (Aravena et al. 2002) podría estar limitada entre otros factores por la menor disponibilidad de luz que tienen las plántulas, debido a la densa cobertura de B. patagonica .

Por lo general, sitios de matorral anegado después de la tala o quema del bosque en Chiloé, también son invadidos por musgos del género Sphagnum, principalmente por $S$. magellanicum, que forma cojines entre los arbustos de $B$. patagonica. Las especies de Sphagnum se caracterizan por sus propiedades morfológicas, anatómicas y fisiológicas que generan sustratos orgánicos poco permeables, pobres en nutrientes, ácidos y aislantes del calor (Van Breemen 1995). Estas condiciones de hábitat, producidas por Sphagnum a través de acumulación de materia orgánica, son limitantes del crecimiento de muchas plantas vasculares, manteniendo las condiciones locales de alta disponibilidad de luz y saturación de humedad que favorecen su propia regeneración (Van Breemen 1995). No obstante, algunos autores (Donoso 1993) indican que Sphagnum sería un buen sustrato para la regeneración en bosques costeros húmedos del sur de Chile, donde Fitzroya cupressoides se asocia con Tepualia stipularis y Pilgerodendron uviferum. En Chiloé, Papic (2000) estudió la colonización de especies arbóreas en sitios abiertos postperturbación por fuego. Sus resultados sugieren que el proceso de colonización de especies arbóreas en estos matorrales secundarios estaría fuertemente restringido a microhábitats elevados por sobre el nivel del suelo, formados por acumulaciones de material leñoso muerto. La colonización por arbustos, en cambio, no parece estar limitada a 
elevaciones del sustrato, por lo que especies arbustivas dominantes de estas áreas sucesionales, serían más tolerantes a las condiciones de anegamiento estacional de los suelos que las especies arbóreas colonizadoras (Papic 2000). La monopolización del sustrato por Baccharis patagonica podría inhibir la continuación del proceso sucesional. La dominancia de especies arbustivas en grandes áreas sucesionales postincendios respondería a una mayor tolerancia de los arbustos al anegamiento estacional de los suelos en comparación con los árboles.

Con estos antecedentes nos planteamos las siguientes hipótesis: la supervivencia de plántulas arbóreas pioneras sombra-intolerantes sería menor bajo cobertura de Baccharis comparado con sitios abiertos entre arbustos. El menor acceso a la luz tendría un efecto negativo en la supervivencia de las plántulas de árboles pioneros sombra-intolerantes. Postulamos, también, que las condiciones de hábitat producidas por los cojines de Sphagnum vivos serían desfavorables para la germinación y supervivencia de plántulas arbóreas. Además, se evaluaron las diferencias en la respuesta de germinación de semillas de especies leñosas pioneras en gradientes de humedad del suelo producidas en forma experimental para representar las variaciones de humedad postperturbación en sitios dominados por matorral.

\section{MATERIALES Y MÉTODOS}

\section{Área de estudio}

El área de estudio está ubicada al noreste de la

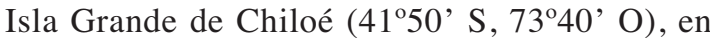
los terrenos de la Estación Biológica "Senda Darwin", comuna de Ancud (Fig. 1). El clima es templado húmedo con fuerte influencia oceánica. La temperatura media anual es $10{ }^{\circ} \mathrm{C}$ y las precipitaciones oscilan entre 2.000 y $2.500 \mathrm{~mm}$ al año (Fig. 2) con un período más seco (aproximadamente un $11 \%$ de la precipitación anual) durante enero y febrero (Di Castri \& Hajek 1976). Se estudió el proceso de regeneración arbórea en planicies de matorral sucesional postincendio, donde la presencia de tocones y troncos semienterrados con marcas de fuego indicaban la presencia de vegetación boscosa antes de la perturbación. Por las condiciones del sitio y las edades de los bosques sucesionales cercanos estimadas por métodos dendrocronológicos convencionales, se puede estimar que estos incendios tuvieron lugar 40-60 años atrás (Aravena et al. 2002).

Se escogieron dos sitios de matorral sucesional, los cuales se caracterizaban por presentar saturación periódica o prolongada de agua, alta frecuencia de especies palustres y cobertura de especies arbustivas bajas como Gaultheria mucronata, Baccharis patagonica, Myrteola nummularia, entre otros. Entre los musgos, destacaba la presencia de cojines de Sphagnum magellanicum, ocupando extensas áreas de suelo entre los arbustos. El sitio 1 presentó una menor frecuencia de Baccharis patagonica (54 versus $60 \%$ ) y de Sphagnum magellanicum (33 versus $52 \%$ ) que el sitio 2 . El suelo fue levemente más ácido en el sitio 2 que en el sitio 1 ( $\mathrm{pH}=4,2$ versus 4,3 , respectivamente) y el contenido hídrico fue mayor en el sitio 2 (5,5 g agua por g suelo seco versus 4,1 g agua por $\mathrm{g}$ suelo seco). La densidad aparente del suelo $\left(0,2 \mathrm{~g} \mathrm{~cm}^{-3}\right)$ no varió entre sitios (M.F. Díaz resultados no publicados). Para evaluar la regeneración natural de especies leñosas en terreno se muestreó las áreas de matorral y el bosque adyacente en cada sitio.

Especies arbóreas y semillas utilizadas en ensayos de terreno e invernadero

En los experimentos se usaron semillas y plántulas de dos especies leñosas pioneras comunes en el bosque siempreverde de la Isla Grande de Chiloé (Aravena et al. 2002). Para los experimentos de supervivencia de plántulas se usaron las especies Embothrium coccineum y Drimys winteri. Embothrium es una especie sombra-intolerante, característica de sitios abiertos, bordes de bosque y campos abandonados (Donoso 1989). Esta especie presenta un alto porcentaje de germinación (> $90 \%$ ) en ensayos de laboratorio y terreno (Figueroa et al. 1996), sus semillas presentan una alta viabilidad y son dispersadas por viento (Figueroa \& Hernández 2001). Debido a la baja capacidad germinativa de muchas especies arbóreas en ensayos previos (Figueroa et al. 1996), se usó esta especie de rápida germinación como modelo en todos los ensayos de terreno y laboratorio. Para los ensayos de 
germinación de semillas en invernadero se usaron también las especies pioneras Nothofagus nitida y Tepualia stipularis.

Regeneración natural de especies leñosas en terreno

Para evaluar la densidad de plántulas $(<50 \mathrm{~cm}$ de alto) en terreno, se muestrearon tres transectos de 100 metros de longitud en cada uno de los dos sitios sucesionales (seis transectos en total). Estos transectos partían en el centro del área de matorral sucesional y terminaban al interior del bosque adyacente, pasando por la transición matorral-bosque. La zona de transición corresponde a unos 30 metros de extensión desde donde comienza el follaje de los árboles, hasta el interior del bosque. Cada 10 metros en cada transecto, se contabilizaron todas las plántulas leñosas presentes en una parcela de $1 \mathrm{~m}^{2}$. En total se muestrearon 24 parcelas en matorral, 18 en la transición matorral-bosque y 18 en el interior del bosque. Los resultados se analizaron estadísticamente mediante un análisis de varianza (ANDEVA) en bloques, considerando los dos sitios como bloques, y el tipo de vegetación (matorral vs. transición o bosque) como los tratamientos.

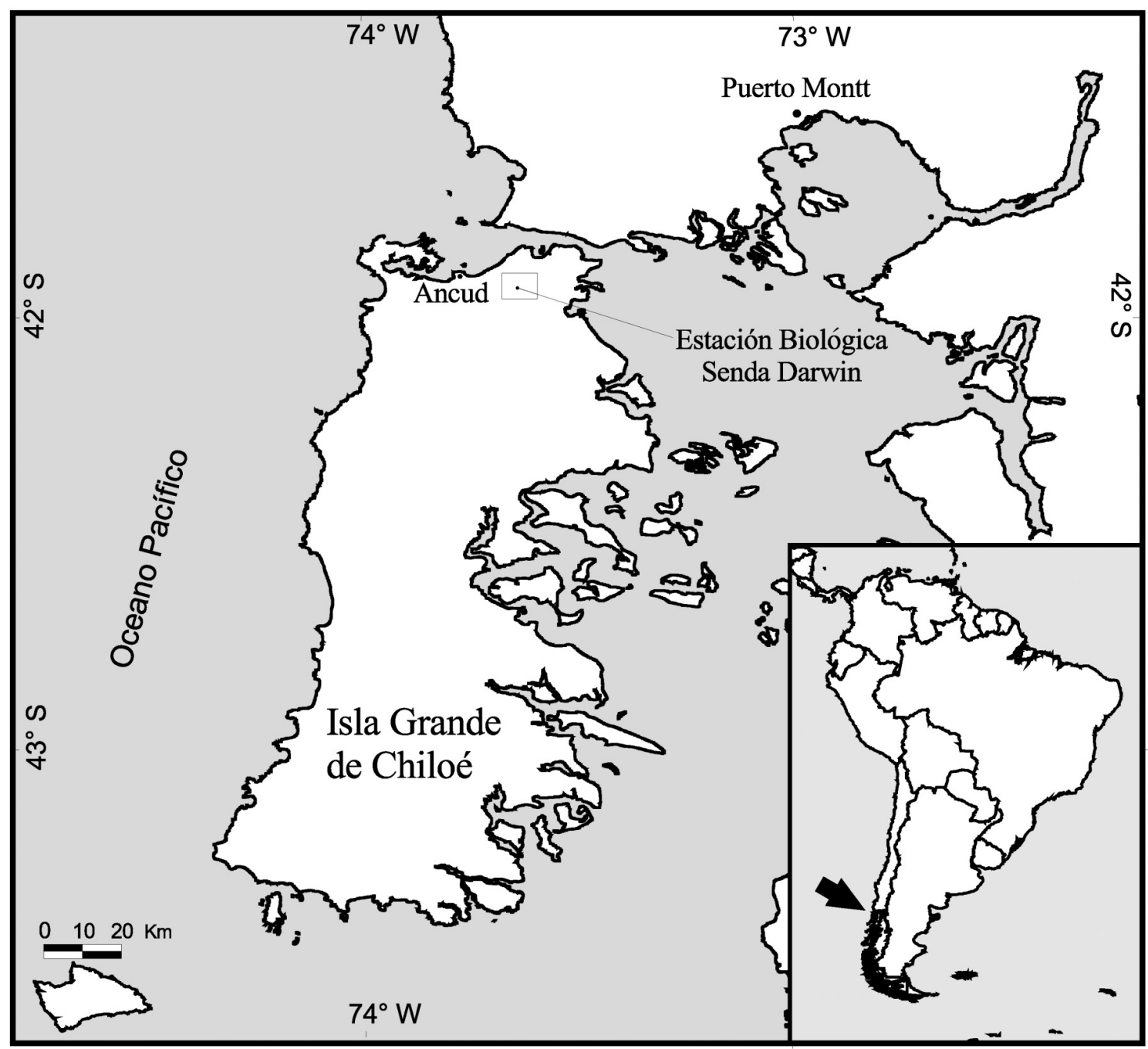

Fig. 1: Sitios de estudio en la Estación Biológica Senda Darwin, en la comuna de Ancud, al norte de la Isla Grande de Chiloé. 


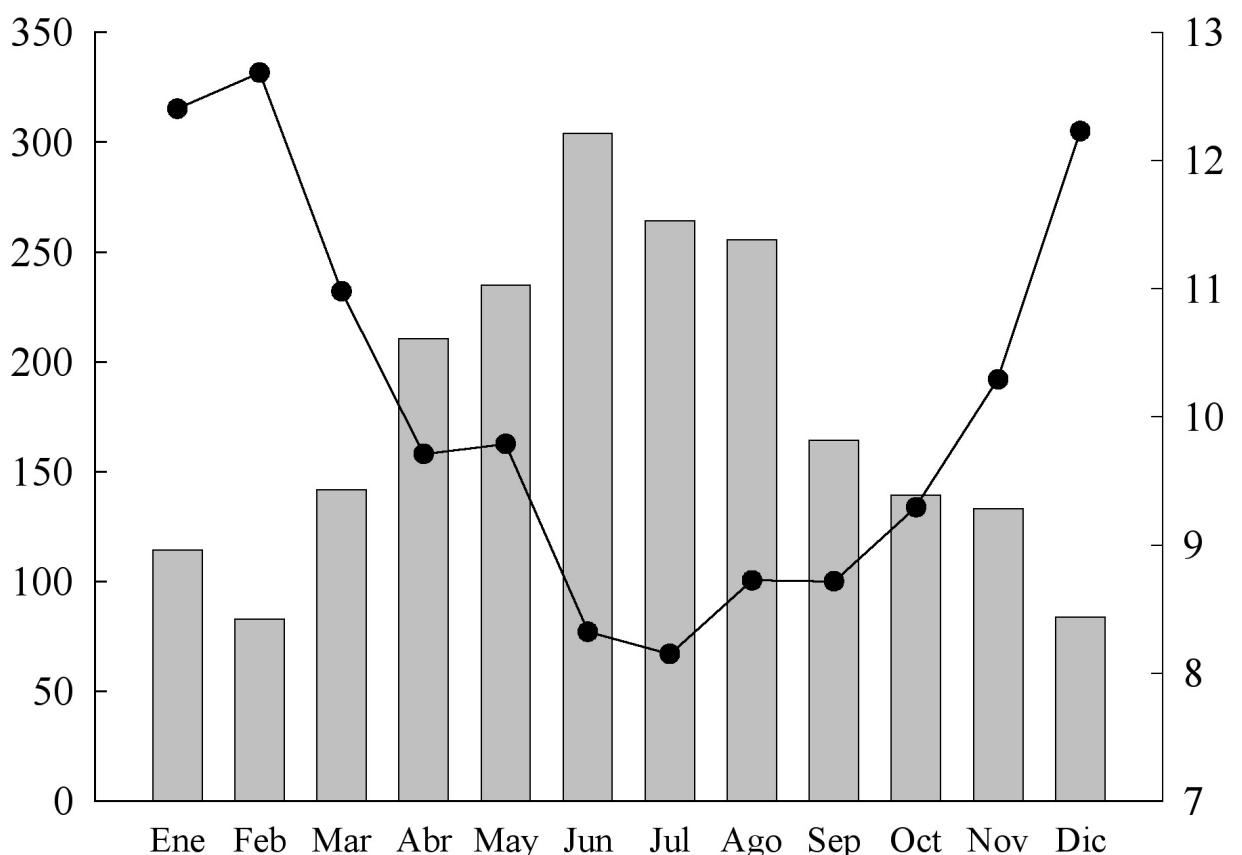

\section{Meses}

Precipitación media

$\longrightarrow$ Temperatura media

Fig. 2: Climograma Estación Biológica Senda Darwin, Chiloé (42 $\left.{ }^{\circ} \mathrm{S}\right)$. Promedios mensuales de precipitación (barras) y temperatura (línea) para un registro de diez años.

Climatic diagram for Senda Darwin Biological Station in Chiloé ( $\left.42^{\circ} \mathrm{S}\right)$. Monthly averages of rainfall (bars) and temperature (line) from 10-year records.

Efecto del anegamiento y sustrato en la germinación de semillas de especies pioneras

Para este experimento se confeccionaron tres cajas de acrílico de 75 × 30 × $15 \mathrm{~cm}$ divididas en seis compartimentos cada una. Tres compartimentos se llenaron con suelo orgánico extraído de los sitios de estudio y otros tres con Sphagnum magellanicum. Las cajas se colocaron en el invernadero de la Estación Biológica Senda Darwin, y se inclinaron $10^{\circ}$ para producir un desnivel que mantenía un suelo más húmedo en el extremo inferior y más seco en el superior. Se regó periódicamente para mantener el nivel del agua en todos los compartimentos. La capacidad de campo del suelo usado en los experimentos es $1,34 \mathrm{~g}$ de agua por $\mathrm{g}$ de suelo seco. Esta se midió una sola vez saturando el suelo colocado en un embudo y midiendo su contenido hídrico con el método gravimétrico una vez que dejaba de escurrir el agua.
Este experimento se repitió en los años 2002 y 2003. El primer año se evaluó la germinación de tres especies arbóreas pioneras (Embothrium coccineum, Nothofagus nitida, Tepualia stipularis) y una especie de arbusto colonizador de lugares abiertos postperturbación (Baccharis patagonica). Todas las semillas utilizadas en este estudio provenían de bosques aledaños, en la Estación Biológica Senda Darwin. Se remojaron las semillas por $24 \mathrm{~h}$ y pasado este tiempo se eliminaron las semillas vanas (las que flotaban).

Se examinó el efecto de los dos tipos de sustrato sobre la geminación: suelo sin Sphagnum magellanicum y suelo con Sphagnum magellanicum. En el caso de E. coccineum se sembraron 110 semillas por compartimento; 83 semillas de $N$. nitida; 100 semillas de $T$. stipularis y 100 semillas de $B$. patagonica, a una profundidad de $1 \mathrm{~cm}$. La única especie que germinó en todos los 
tratamientos fue E. coccineum, por lo que los resultados se muestran solo para esta especie. Para el análisis estadístico se empleó un análisis de varianza de dos vías, donde los factores fueron el tipo de sustrato (Sphagnum versus suelo) y nivel de humedad (anegado, húmedo y relativamente más seco). La variable dependiente fue el porcentaje de semillas germinadas. El tiempo de medición fue el tiempo de germinación promedio para cada especie (90-120 días) (Figueroa et al. 1996).

El año 2003 se repitió el experimento pero solo sobre el sustrato suelo y con las especies pioneras $E$. coccineum, $N$. nitida y $D$. winteri. Se sembraron 100 semillas de cada especie por compartimento. Esta vez se aumentó a $15^{\circ}$ la inclinación de las cajas para lograr una diferencia estadísticamente significativa en los niveles de humedad del suelo entre los compartimentos extremos. Se encontró diferencias significativas entre el nivel más seco y el más húmedo en el porcentaje de humedad del suelo $\left(\mathrm{F}_{2,15}=41,97 ; \mathrm{P}<0,001\right)$. El compartimento más bajo (que simulaba el nivel de napa freática más elevado; o suelo anegado) presentó un $113 \%$ de la capacidad de campo; el del medio (estado intermedio o húmedo) presentó un $107 \%$ de la capacidad de campo; y el último compartimento (relativamente más seco), un $85 \%$ de la capacidad de campo del suelo. En este experimento no hubo germinación de $D$. winteri, solo de E. coccineum y N. nitida.

Efecto de la cobertura de Baccharis patagonica en la supervivencia de plántulas leñosas

Para evaluar el efecto de la cobertura del follaje de Baccharis patagonica en la supervivencia de plántulas pioneras del bosque, se manipuló la cobertura de Baccharis. Se removió la mitad del dosel de 10 arbustos seleccionados al azar en el matorral, y se evaluó la supervivencia de plántulas trasplantadas bajo las dos condiciones (con y sin remoción de dosel) mediante un análisis de curvas de supervivencia Log-rank (Fox 1993). Se usaron plántulas de edad conocida (originadas de semillas colectadas en bosques aledaños a los sitios de estudio) de las especies Drimys winteri (1 año, entre 3-5 cm de altura) y Embothrium coccineum (2 años, entre 15-30 cm de altura), las cuales fueron mantenidas en el vivero por ese tiempo y luego aclimatadas. Se usaron 10 plántulas por tratamiento (con y sin remoción de dosel), $\mathrm{n}=$ 20 plántulas por especie y por sitio (40 plántulas en cada sitio de las especies $D$. winteri y E. coccineum). Los trasplantes se realizaron en el mes de agosto de 2002, a una profundidad de $10 \mathrm{~cm}$. El período de evaluación fue de 21 meses. El primer año se registró la supervivencia cada mes y luego los períodos de evaluación se fueron distanciando.

\section{Efecto de la cobertura de Sphagnum magellanicum}

Se trasplantaron plántulas leñosas sobre cojines de Sphagnum vivo y sobre sustrato donde se removió un volumen de $\sim 1.000 \mathrm{cc}$ de musgo, se secó al aire y se repuso en el mismo lugar. Este experimento tuvo como objeto evaluar si la arquitectura natural del musgo formando cojines compactos daba cuenta de las propiedades adversas para el establecimiento de plantas leñosas. La sobrevivencia se evaluó con un análisis de curvas de supervivencia Log-rank (Fox 1993) por un período de 19 meses, comenzando en septiembre de 2002. En los trasplantes se usaron 10 plántulas por tratamiento (Sphagnum vivo y muerto) de edad conocida (originadas de semillas) de las especies pioneras Drimys winteri y Embothrium coccineum. El número total de plántulas por especie fue de $20, n=40$ plántulas por sitio.

\section{Diferencias ambientales entre microhábitats}

Se evaluó si los tratamientos con y sin remoción de dosel de Baccharis patagonica diferían en intensidad lumínica al nivel del suelo. Para esto se midió la intensidad de luz con un luxímetro en cada punto donde se hicieron los ensayos de supervivencia. También se midió la temperatura del suelo (mediante un termómetro convencional, a $10 \mathrm{~cm} \mathrm{de}$ profundidad) y del sustrato Sphagnum en los casos de ensayos de supervivencia sobre musgos. Estas mediciones se realizaron un mismo día en el lapso de una hora en los dos sitios de estudio. Se utilizó la prueba no paramétrica de Scheirer-Ray-Hare, una extensión a la prueba de Kruskal-Wallis (Sokal \& Rohlf 1995) para evaluar si existían diferencias entre microhábitat. 


\section{RESULTADOS}

Regeneración natural de especies leñosas en terreno

En condiciones naturales, la regeneración arbórea en los dos sitios de matorral secundario fue muy baja $\left(<0,5\right.$ plántulas $\left.\mathrm{m}^{-2}\right)$. Se observó un incremento en la densidad de plántulas arbóreas al aproximarse al borde del bosque (Fig. 3). En el centro del matorral sucesional, la densidad promedio $( \pm$ EE) de plántulas fue $0,3 \pm 0,2$ plántulas $\mathrm{m}^{-2}$, y en la transición matorral-bosque, la densidad aumentó a 2,6 $\pm 1,1$ plántulas $\mathrm{m}^{-2}$. En el interior del bosque se encontró la mayor densidad de plántulas, 7,2 $\pm 1,1$ plántulas $\mathrm{m}^{-2}$. Estas diferencias fueron estadísticamente significativas $\left(\mathrm{F}_{2,50}=14,09 ; \mathrm{P}<0,001\right)$. En el sitio 1 , todas las plántulas registradas en el matorral sucesional fueron de la conífera sombraintolerante Pilgerodendron uviferum (Tabla 1). Datos genéticos usando RAPDs indican que estos son individuos derivados de semillas (A. Gabriel resultados no publicados).

\section{Germinación en gradientes de humedad}

En el ensayo del año 2002 no se estableció un gradiente experimental de humedad que representara diferencias significativas entre los tres compartimentos (bajo, medio y alto), de manera que el experimento solo evaluó diferencias entre sustrato suelo versus Sphagnum. La única especie que germinó en los experimentos fue E. coccineum. La germinación de E. coccineum fue significativamente mayor sobre el sustrato de musgo Sphagnum (61\%) que sobre suelo $(41 \%)\left(\mathrm{F}_{1,12}=28,92 ; \mathrm{P}<0,001\right)$ (Fig. 4).

En el año 2003 se logró establecer un gradiente de humedad estadísticamente significativo entre los tres compartimentos $\left(\mathrm{F}_{2,15}\right.$ = 41,97; $\mathrm{P}<0,001)$. Sin embargo, no hubo diferencias en el porcentaje de germinación entre los tratamientos de humedad para ninguna de las especies que germinaron (E. coccineum y $N$. nitida). Las semillas de E. coccineum obtuvieron en promedio un $76 \%$ de germinación, mientras que las de N. nitida un $10 \%$.

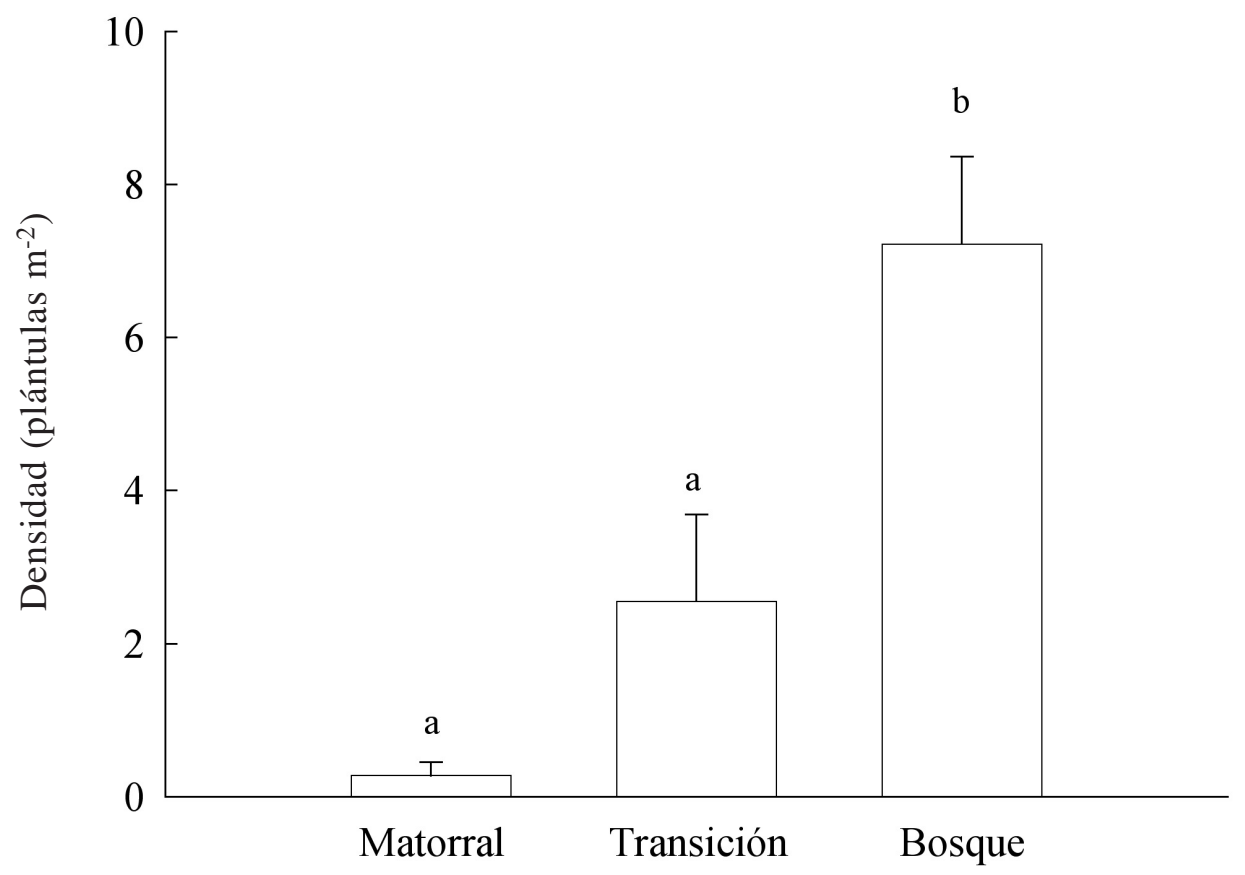

Fig. 3: Densidad de plántulas arbóreas en matorral sucesional, transición matorral-bosque y bosque secundario. Promedio del número de plántulas $\mathrm{m}^{-2}$ en seis transectos con 18 parcelas por cada tipo de vegetación (matorral, transición y bosque). Letras distintas indican diferencias estadísticamente significativas $(\mathrm{P}<0,05)$.

Seedling density in successional shrublands, in shrubland-forest transition, and within adjacent secondary forest. Average number of seedlings $\mathrm{m}^{-2}$ in six transects with 18 plots for each vegetation zone (shrubland, transition and forest). Different letters indicate statistically significant differences $(\mathrm{P}<0.05)$. 


\section{TABLA 1}

Especies de plántulas observadas en matorral, transición matorral-bosque y bosque, en ambos sitios de estudio combinados

Seedling species recorded in successional shrublands, in shrubland-forest transition, and within adjacent secondary forest, in both study sites combined

\begin{tabular}{lccc}
\hline Especie & Matorral abierto & $\begin{array}{c}\text { Número de plántulas } \\
\text { Transición matorral-bosque }\end{array}$ & Bosque secundario \\
\hline Amomyrtus meli & 0 & 5 & 44 \\
Caldcluvia paniculata & 0 & 4 & 21 \\
Drimys winteri & 0 & 11 & 18 \\
Eucryphia cordifolia & 0 & 4 & 1 \\
Gevuina avellana & 0 & 6 & 0 \\
Myrceugenia parvifolia & 0 & 2 & 1 \\
Nothofagus nitida & 0 & 6 & 1 \\
Pilgerodendron uviferum & 6 & 0 & 0 \\
Podocarpus nubigena & 0 & 1 & 10 \\
Saxegothaea conspicua & 0 & 0 & 3 \\
Tepualia stipularis & 0 & 7 & 23 \\
Weinmannia trichosperma & 0 & 0 & 4 \\
Total & & 46 & 126 \\
\hline
\end{tabular}

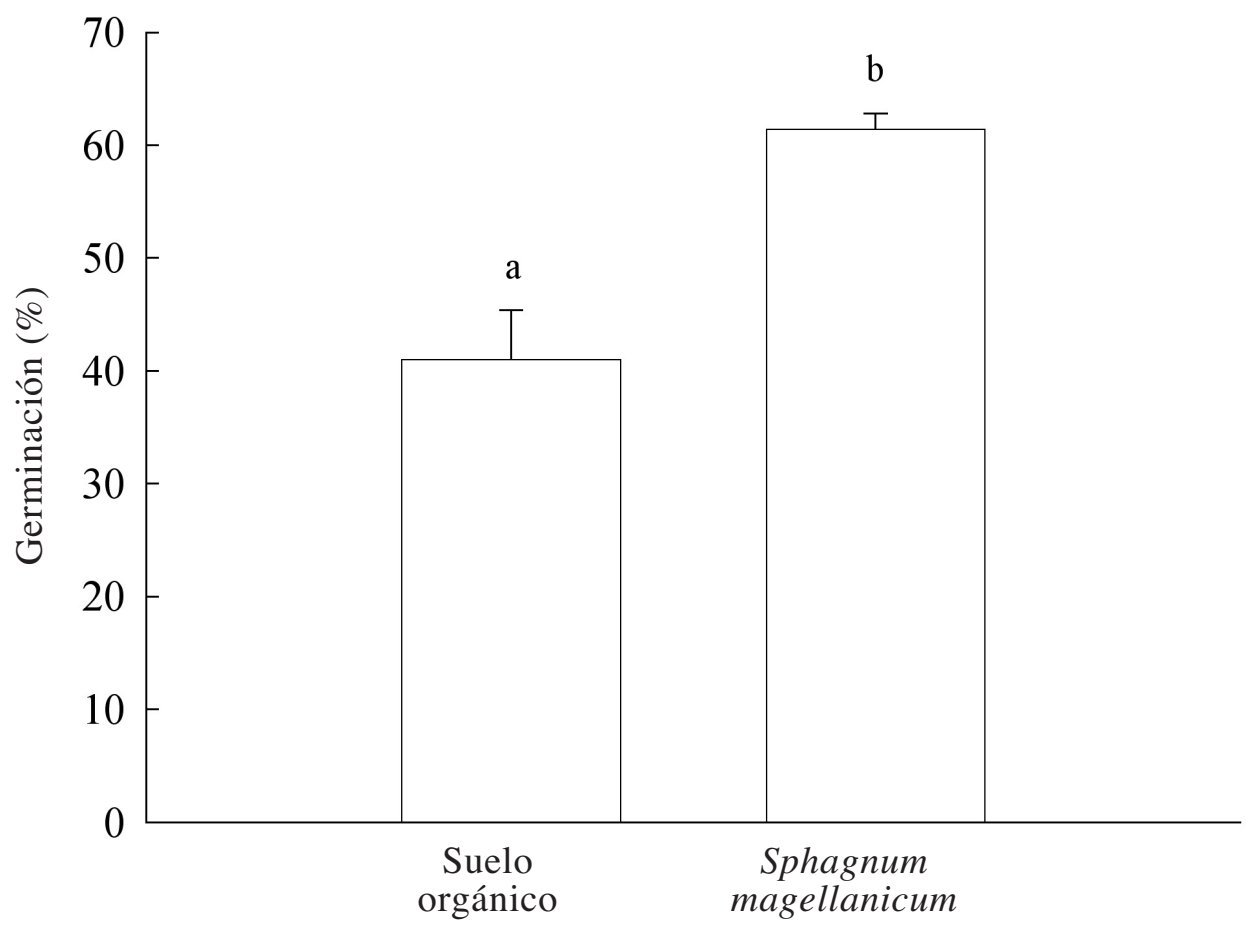

Fig. 4: Germinación de Embothrium coccineum sobre dos sustratos: suelo y Sphagnum magellanicum. Valores promedio \pm EE. Las letras distintas indican diferencias estadísticamente significativas.

Seed germination of Embothrium coccineum on two substrates: soil and cushions of Sphagnum magellanicum. Mean values \pm SE. Different letters indicate statistically significant differences. 
Supervivencia de plántulas de Drimys winteri en matorral sucesional

La supervivencia de $D$. winteri no fue afectada por la cobertura del dosel de Baccharis. En ambos sitios no hubo diferencias significativas en la supervivencia bajo Baccharis con o sin dosel. La supervivencia fue mayor en el sitio 1 (85\%, basado en el total de plántulas de $D$. winteri, sin distinción de tratamiento de luz) que en el sitio $2(35 \%)$. En el sitio 2 se observó mayor mortalidad de plántulas en ausencia de dosel de Baccharis $(\mathrm{L}=1,84 ; \mathrm{P}=0,07 ; \mathrm{n}=20$, análisis de curvas de supervivencia Log-rank), la diferencia fue marginalmente significativa. En ambos sitios, contrariamente a lo esperado, la tendencia, aunque solo marginalmente significativa, fue de mayor supervivencia de plántulas de Drimys bajo la protección de la cobertura de Baccharis. La cobertura de Baccharis en ambos sitios fue 60 y $54 \%$ (Díaz 2004). La mayor mortalidad de plántulas se observó durante el período estival y a comienzos del otoño austral (febrero-abril 2002), cuando las precipitaciones son menores que en otras estaciones del año (Fig. 2).

No hubo efectos significativos al comparar la supervivencia de plántulas sobre Sphagnum vivo o muerto $(\mathrm{L}=1,38 ; \mathrm{P}=0,17 ; \mathrm{n}=40$, prueba realizada para los dos matorrales). Nuevamente, la sobrevivencia fue menor en el sitio $2(35 \%)$ que en el sitio $1(65 \%)$. Como en el caso del tratamiento bajo Baccharis, la mayor mortalidad ocurrió durante el período estival y comienzos de otoño.

Supervivencia de plántulas de Embothrium coccineum en matorral sucesional

No hubo efecto de los tratamientos de remoción de dosel sobre la supervivencia de plántulas de la especie pionera E. coccineum (L $=1,12 ; \mathrm{P}=0,26 ; \mathrm{n}=20$ para el sitio $1, \mathrm{y} \mathrm{L}=$ $1,07 ; \mathrm{P}=0,29 ; \mathrm{n}=20$ para el sitio 2$)$. La mortalidad fue alta (50-90\% en algunos tratamientos) en ambos sitios.

La supervivencia de plántulas de Embothrium tampoco mostró diferencias significativas entre Sphagnum vivo y muerto (L $=-0,11 ; \mathrm{P}=0,91 ; \mathrm{n}=20$ para el sitio $1, \mathrm{y} \mathrm{L}=-$ $1,45 ; \mathrm{P}=0,15 ; \mathrm{n}=20$ para el sitio 2$)$. En este ensayo la mortalidad sobre Sphagnum fue menor que sobre suelo bajo Baccharis $(10 \%$ de mortalidad sobre Sphagnum, versus $70 \%$ sobre el suelo). Considerando solo el sustrato de establecimiento de las plántulas (suelo vs. Sphagnum), y sumado los datos de ambos matorrales, se encontraron diferencias significativas en la supervivencia de $E$. coccineum $(\mathrm{L}=3,47 ; \mathrm{P}<0,001 ; \mathrm{n}=80)$ entre los sustratos (Fig. 5B).

\section{Condiciones de luz y temperatura de los trata- mientos}

Existen diferencias significativas en la luz que reciben las plántulas en microhábitat sin dosel y bajo dosel de Baccharis, y también en sitios abiertos sobre Sphagnum. La diferencia entre tratamientos fue significativa $(\mathrm{H}=13,79 ; \mathrm{gl}=2$; $\mathrm{P}=0,001$, prueba no paramétrica de ScheirerRay-Hare). En promedio para los dos sitios, la intensidad lumínica bajo Baccharis con remoción de dosel fue: 5.013,5 \pm 949 lux, bajo Baccharis intacto: $2.071,5 \pm 308$ lux, y sobre cojines de Sphagnum se registró: 6.147,5 \pm 1.082 lux. Las plántulas bajo Baccharis sin dosel están igualmente expuestas que las plantas que crecen sobre cojines de Sphagnum situados en espacios abiertos entre arbustos. La temperatura difirió entre los cojines de Sphagnum y el suelo bajo Baccharis, con o sin cobertura de dosel $(\mathrm{H}$ $=8,046 ; \mathrm{gl}=2 ; \mathrm{P}=0,018$, prueba no paramétrica de Scheirer-Ray-Hare). El sustrato Sphagnum mostró mayores temperaturas que el sustrato suelo en 0,5 a $1{ }^{\circ} \mathrm{C}$.

\section{DISCUSIÓN}

En el hemisferio norte, los estudios de colonización de plántulas de árboles y arbustos en praderas abandonadas después de la eliminación del bosque (De Steven 1991a, 1991b, Gill \& Marks 1991, Callaway 1992) apuntan, en su mayoría, a evaluar los mecanismos de sucesión propuestos por Connell \& Slatyer (1977). Estos tres modelos (tolerancia, facilitación e inhibición) pueden actuar durante la sucesión como hipótesis no excluyentes (Pickett et al. 1987). En el bosque templado del sur de Chile, la mayoría de los estudios de regeneración arbórea se han realizado en el interior de bosques primarios y secundarios (Armesto \& Figueroa 1987, Lusk 1995, Christie \& Armesto 2003). Muy pocos 
trabajos han evaluado la regeneración leñosa en sitios de matorral secundario (e.g., Papic 2000, Aravena et al. 2002). Tanto los estudios en bosques como en áreas sucesionales han notado la importancia de la regeneración arbórea sobre detritus leñoso.

También se ha evaluado la supervivencia de plántulas leñosas con distintos requerimientos lumínicos (Figueroa \& Lusk 2001), comparando la supervivencia bajo dosel y en claros de bosque. Sin embargo, no se ha abordado el tema de los mecanismos de regeneración arbórea en sitios de matorral secundario, donde las condiciones de anegamiento estacional, la abundancia del arbusto Baccharis patagonica y la presencia del musgo Sphagnum podrían estar limitando la germinación y supervivencia de plántulas leñosas. Este tipo de estudios son
(A)

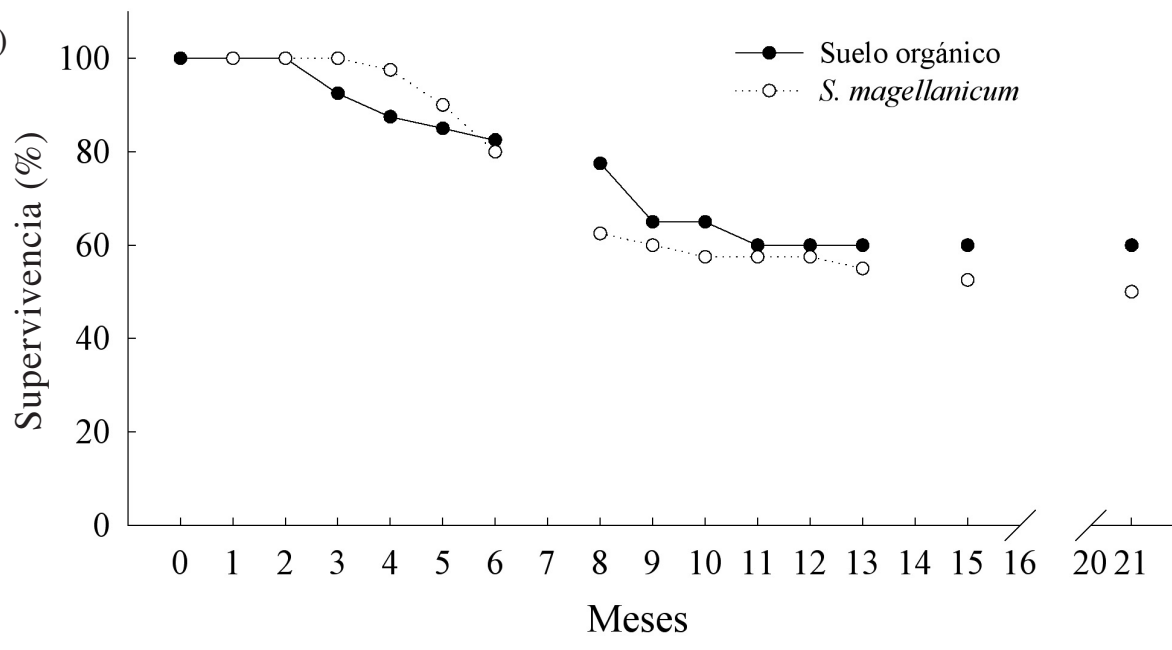

(B)

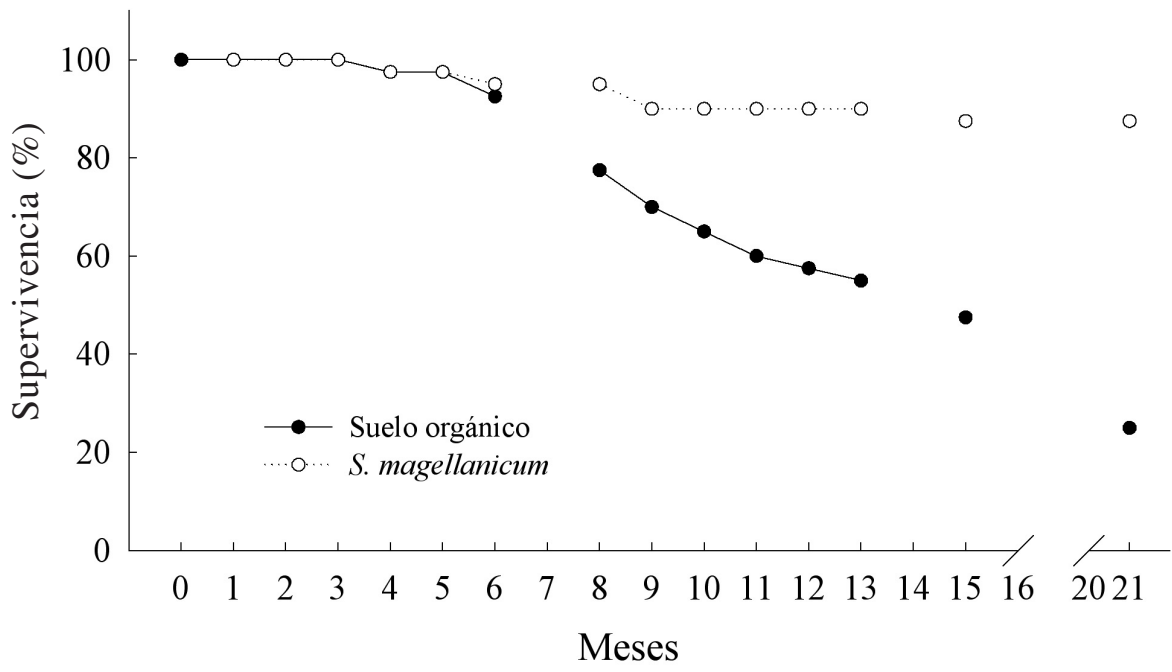

Fig. 5: Curvas de supervivencia de plántulas de Drimys winteri (A) y Embothrium coccineum (B) plantadas sobre el suelo (círculos negros) y sobre cojines de Sphagnum magellanicum (círculos abiertos). La interrupción en los datos indica que durante esos meses no se obtuvieron registros de supervivencia. No hubo efectos de sustrato en Drimys $(\mathrm{L}=-0,86 ; \mathrm{P}=0,39)$, pero si en Embothrium $(\mathrm{L}=3,47 ; \mathrm{P}<0,001)$.

Survival curves of Drimys winteri (A) and Embothrium coccineum (B) seedlings planted on soil (closed circles) and on cushions of Sphagnum magellanicum (open circles). Interruptions of data indicate no records of survival for those months. There were no significant effects of substrate for Drimys $(\mathrm{L}=-0.86, \mathrm{P}=0.39)$, but there significant differences for Embothrium $(\mathrm{L}=3.47, \mathrm{P}<0.001)$. 
relevantes debido al significativo incremento de las áreas de bosques quemados, talados y cubiertos por matorrales en el sur de Chile. En Chiloé los matorrales han incrementado en superficie, de un $2,7 \%$ de la superficie total comunal (Ancud) en 1985, a un 7,4\% en 1999 (Díaz 2004). La baja regeneración natural en estos sitios los hace susceptibles a mayores perturbaciones, por drenaje artificial, y a la reforestación con especies exóticas como Eucalyptus, que pueden alterar las condiciones hidrológicas del área.

La regeneración natural (Fig. 3), muestra una mayor densidad de plántulas concentrada, en el suelo del interior del bosque y casi nula regeneración en los matorrales postfuego. Este resultado documenta que el progreso de la sucesión hacia la condición representada por el bosque original se encuentra severamente impedida por las nuevas características del sitio perturbado, incluyendo la cobertura de Baccharis, el anegamiento y la invasión del musgo Sphagnum. Estas condiciones postfuego representan una vuelta a los ambientes sin bosque, dominados por plantas de turbera, que caracterizaron los sitios postglaciación hace más de 10.000 años, como documentan estudios palinológicos de los sitios bajos de Chiloé para fines del último Glacial ( Villagrán et al. 1986, Villagrán 1990a, 1990b, Moreno \& León 2003, Abarzúa et al. 2004, Moreno 2004). A través de los experimentos de germinación y trasplante, esperábamos dilucidar las limitantes actuales de la recuperación del bosque en estos sitios.

Los ensayos de germinación de semillas en gradientes experimentales de humedad del sustrato no fueron conclusivos debido a la escasa germinación, pero en las especies que germinaron no hubo efectos de la humedad del sustrato. Aparentemente, ni Embothrium coccineum ni Nothofagus nitida tendrían problemas de germinación en sustratos con alta humedad. Sin embargo, es posible que las condiciones en el experimento difieran de las condiciones de anegamiento en terreno, donde muchas veces la napa alcanza la superficie, formando grandes posas. En nuestro experimento, Embothrium coccineum tuvo $68 \%$ de germinación en el compartimento anegado, Nothofagus nitida solo un $8 \%$, lo que indica una mayor capacidad de Embothrium de germinar en suelos saturados de humedad.
Supervivencia de plántulas leñosas bajo cobertura de Baccharis patagonica

En los ensayos de supervivencia, la sombra de $B$. patagonica no fue una limitante para la supervivencia de plántulas de la especie pionera Drimys winteri. A pesar de ser una especie de tolerancia intermedia, según Donoso (1989), la supervivencia de las plántulas fue mayor, aunque marginalmente significativa, bajo el dosel intacto del arbusto. Según Figueroa \& Lusk (2001), los requerimientos lumínicos de $D$. winteri son de $8,8 \%$ de apertura promedio del dosel, porcentaje bastante bajo comparado con los requerimientos lumínicos de Embothrium coccineum y Baccharis patagonica $(25,6$ y $76,5 \%$ respectivamente). En el caso de $D$. winteri, las condiciones de cobertura rala del matorral secundario representarían un ambiente desfavorable para el establecimiento de esta especie, caracterizado por un porcentaje promedio de apertura de dosel de 30 \% (Díaz 2004). La mayor mortalidad de plántulas se observó en el verano austral, cuando las precipitaciones disminuyen, ya que solo un $17 \%$ de la precipitación anual cayó durante estos tres meses (Fig. 2). Febrero además es el mes más seco (con aproximadamente un $3 \%$ de la precipitación anual, datos de la estación meteorológica "Senda Darwin"). El desecamiento de los sustratos abiertos de matorral secundario en los meses estivales podría contribuir fuertemente a la mortalidad de estas plántulas, a pesar del anegamiento de los meses invernales más lluviosos.

Tampoco hubo efecto de sombra del dosel de Baccharis en la supervivencia de plántulas de Embothrium coccineum. Nuestros resultados indican que esta especie sería capaz de establecerse en estos matorrales sucesionales tanto bajo como fuera de la cobertura de los arbustos. Aunque no podemos aceptar ni rechazar la hipótesis de menor supervivencia bajo dosel de Baccharis, las tendencias indican que el efecto de desecación estival tendría consecuencias más serias como limitante del reclutamiento que el efecto de sombreamiento. Es posible que la sombra de arbustos favorezca en alguna medida la supervivencia, como ocurre en el caso de Drimys, al reducir la desecación de verano. 
Supervivencia de plántulas sobre cojines de Sphagnum magellanicum

La supervivencia de Drimys winteri sobre cojines de Sphagnum vivo o muerto tampoco presentó diferencias estadísticamente significativas. El musgo seco y puesto sobre el suelo volvió a hidratarse y, a pesar de perder su compactación natural en forma de cojín, no tuvo efecto sobre la supervivencia de las plántulas. En el sitio 2, el $40 \%$ de las plántulas de $D$. winteri sobre musgos desaparecieron bajo el crecimiento de Sphagnum o murieron, en el sitio 1, el porcentaje de mortalidad fue menor (25\%). El crecimiento de Sphagnum por sobre las plántulas también se observó en el tratamiento bajo Baccharis, la mortalidad de Drimys fue menor (10 y $25 \%$, sitios 1 y 2 respectivamente), por lo que el efecto del crecimiento de Sphagnum podría ser relevante en plazos más largos. En algunos puntos Sphagnum invadió y cubrió completamente las plántulas de Drimys que tenían un tamaño inicial de 3-5 cm.

La supervivencia de Embothrium coccineum fue significativamente mayor sobre cojines de Sphagnum que en el suelo bajo los arbustos de Baccharis (Fig. 5). Este resultado, aunque no fue central al estudio planeado, junto con la mayor germinación de E. coccineum sobre sustratos de Sphagnum en invernadero (Fig. 4), nos sugiere que la invasión de este musgo en zonas de matorral secundario favorecería la regeneración de esta especie. Los cojines de Sphagnum se caracterizan por su capacidad de retener agua en el interior de sus tejidos, por lo que el crecimiento de plántulas sobre este musgo no necesariamente sería afectado por anegamiento en la medida que lo son en el suelo saturado. En consecuencia, Sphagnum podría actuar como especie facilitadora para el establecimiento de especies como E. coccineum en el matorral sucesional, aunque en el mismo sitio podría inhibir el establecimiento de otras especies. Así, los mecanismos de facilitación e inhibición actuarían simultáneamente en una misma etapa de la sucesión (Pickett et al. 1987).

No podemos concluir que los factores evaluados en este trabajo sean responsables de la limitada regeneración arbórea en matorrales sucesionales. Por el contrario, Sphagnum al menos, podría facilitar la regeneración de la especie pionera E. coccineum. Otros factores limitantes, alternativos o adicionales a los ya citados, que explicarían el estancamiento sucesional podrían ser la limitada dispersión de semillas desde el bosque a los sitios abiertos y la baja disponibilidad de sustratos elevados por sobre el suelo anegado. Debido a que la distancia de dispersión de semillas de los árboles dominantes del bosque de Chiloé es limitada (Armesto et al. 2001), los cambios más rápidos en la composición de especies ocurrirían en los bordes. Adicionalmente, estudios de la avifauna han mostrado que el matorral de Baccharis patagonica no es atractivo para aves dispersoras de semillas, ni como alimento, ni como sitios percha (Armesto et al. 2004). Así la dispersión de semillas a sitios abiertos es baja. Por otro lado, la baja densidad de detritus leñoso en los sitios de matorral, luego de incendios (Carmona et al. 2002), podría estar limitando el establecimiento de plántulas arbóreas, debido a la ausencia de micrositios elevados. Especies sombra intolerantes de los bosques de Chiloé, tales como Drimys winteri, Nothofagus nitida, Tepualia stipularis, Eucryphia cordifolia, Pseudopanax laetevirens y Weinmannia trichosperma, se establecen con mayor frecuencia sobre detritus leñoso (Christie \& Armesto 2003) y la supervivencia de plántulas sobre detritus leñoso en sitios sucesionales es más alta que sobre suelo desnudo (Papic 2000).

Por el alto porcentaje de germinación que presentó Embothrium coccineum tanto en el suelo como sobre los cojines de Sphagnum, su rápido crecimiento que evita que sea cubierto por Sphagnum, y su respuesta favorable en los ensayos de supervivencia, nos lleva a proponer esta especie para planes de restauración en sitios de matorral sucesional, donde el anegamiento y la falta de sustratos elevados podría estar limitando fuertemente el proceso de regeneración arbórea. La presencia de una densidad creciente de árboles de Embothrium en sitios de matorral sucesional podría contribuir a mejorar el drenaje del sitio a través de su evapotranspiración, favoreciendo el progreso de la sucesión.

Por otro lado, en estos sitios sucesionales los bosques no solo han sido quemados y madereados, sino que en la mayoría de los casos su recuperación ha estado fuertemente limitada por la continua presión ganadera. El pisoteo, ramoneo, y compactación del suelo puede haber 
contribuido al estancamiento sucesional que se observa. Se requieren seguimientos de las plántulas que logran establecerse en el matorral, ya que no sabemos si estas plántulas en el matorral sobrevivirán las repetidas condiciones de anegamiento invernal y sequía estival. También se sugiere evaluar el reclutamiento de semillas de mayor tamaño (como es el caso de $E$. coccineum) en ensayos de terreno directamente en el suelo o sobre Sphagnum, ya que estas semillas grandes presentan un menor grado de dependencia de sustratos de detritus leñoso grueso para su reclutamiento (Lusk 1995, Christie \& Armesto 2003).

\section{AGRADECIMIENTOS}

Este trabajo fue posible gracias al financiamiento de CONICYT (beca de doctorado y apoyo de tesis doctoral), a la beca de la Universidad de Chile PG/17/02 y a la Beca de Doctorado del proyecto Milenio (P02051-F). Agradecemos también el apoyo de FONDAP-FONDECYT 1501-0001 al Centro de Estudios Avanzados en Ecología y Biodiversidad durante la preparación del manuscrito. A Felipe Hinojosa, Olga Barbosa, Rafael Guevara y Mariela Núñez por su ayuda en terreno. Al guardaparque de la Estación Biológica Senda Darwin, Emer Mancilla, y a Juan Vidal por su ayuda en la colecta de semillas y en las actividades del vivero. Esta es una contribución al programa de investigación de la Estación Biológica Senda Darwin, Chiloé.

\section{LITERATURA CITADA}

ABARZÚA AM, C VILLAGRÁN \& PI MORENO (2004) Deglacial and postglacial climate history in eastcentral Isla Grande de Chiloé, southern Chile ( $43^{\circ}$ S). Quaternary Research 62: 49-59.

ARAVENA JC (1991) Análisis de la estructura y la flora de los bosques de Chiloé continental e insular. Tesis de Magíster en Ciencias, mención Botánica, Facultad de Ciencias, Universidad de Chile, Santiago, Chile. 98 pp.

ARAVENA JC, MR CARMONA, CA PÉREZ \& JJ ARMESTO (2002) Changes in tree species richness, stand structure and soil properties in a successional chronosequence of forest fragments in northern Chiloé Island, Chile. Revista Chilena de Historia Natural 75: 339-360.

ARMESTO JJ, I DÍAZ, C PAPIC \& M WILLSON (2001) Seed rain of fleshy and dry propagules in different habitats in the temperate rainforests of Chiloé Island, Chile. Austral Ecology 26: 311-320.
ARMESTO JJ \& J FIGUEROA (1987) Stand structure and dynamics in the rain forest of Chiloé Archipelago, Chile. Journal of Biogeography 14: 367-376.

ARMESTO JJ, M WILLSON, I DÍAZ \& S REID (2004) Ecología del paisaje rural de la Isla de Chiloé: diversidad de especies de aves en fragmentos de bosques nativos. En: Smith-Ramírez C, JJ Armesto \& C Valdovinos (eds) Historia, ecología y biodiversidad de los bosques de la cordillera de la Costa de Chile: 585-599. Editorial Universitaria, Santiago, Chile.

CALLAWAY RM (1992) Effect of shrubs on recruitment of Quercus douglasii and Quercus lobata in California. Ecology 73: 2118-2128.

CARMONA MR, JJ ARMESTO, JC ARAVENA \& CA PÉREZ (2002) Coarse woody debris biomass in successional and primary temperate forests in Chiloé Island, Chile. Forest Ecology and Management 164: 265-275.

CHRISTIE DA \& JJ ARMESTO (2003) Regeneration microsites and tree species coexistence in temperate rain forests of Chiloé Island, Chile. Journal of Ecology 91: 776-784

CONNELL JH \& RO SLATYER (1977) Mechanisms of succession in natural communities and their role in community stability and organization. American Naturalist 111: 1119-1144.

DE STEVEN D (1991a) Experiments on mechanisms of tree establishment in old-field succession: seedling survival and growth. Ecology 72: 1076-1088.

DE STEVEN D (1991b) Experiments on mechanisms of tree establisment in old-field succession: seedling emergence. Ecology 72: 1066-1075.

DI CASTRI F \& ER HAJEK (1976) Bioclimatología de Chile. Editorial de la Universidad Católica de Chile, Santiago, Chile. 128 pp.

DÍAZ MF (2004) Limitantes biológicas e hidrológicas de la sucesión secundaria en bosques de Chiloé. Tesis de Doctorado en Ciencias, mención en Ecología y Biología Evolutiva, Facultad de Ciencias, Universidad de Chile, Santiago, Chile. $141 \mathrm{pp}$.

DONOSO C (1989) Antecedentes básicos para la silvicultura del tipo forestal siempreverde. Bosque (Chile) 10: 37-53.

FIGUEROA J, JJ ARMESTO \& JF HERNÁNDEZ (1996) Estrategias de germinación y latencia de semillas en especies del bosque templado de Chiloé, Chile. Revista Chilena de Historia Natural 69: 243-251.

FIGUEROA J \& C LUSK (2001) Germination requirements and seedling shade tolerance are not correlated in a Chilean temperate rain forest. New Phytologist 152: 483-489.

FIGUEROA JA \& JF HERNÁNDEZ (2001) Effect of a gap and canopy condition on seed germination in species of a temperate rainforest in the Archipelago de Chiloé, Chile. Ecología Austral 11: 39-47.

FOX GA (1993) Failure-time analysis: emergence, flowering, survivorship, and other waiting times. En: Scheiner SM \& YJ Gurevitch (eds) Design and analysis of ecological experiments: 253-289. Chapman and Hall, New York, New York, USA.

GILL DS \& PL MARKS (1991) Tree and shrub seedling colonization of old fields in central New York. Ecological Monographs 61: 183-205.

LUSK C (1995) Seed size, establishment sites and species coexistence in a Chilean rain forest. Journal of Vegetation Science 6: 249-256.

MORENO PI \& AL LEÓN (2003) Abrupt vegetation changes during the last glacial to Holocene transition in mid-latitude South America. Journal of Quaternary Science 18: 787-800. 
MORENO PI (2004) Millennial-scale climate variability in northwest Patagonia over the last 15000 yr. Journal of Quaternary Science 19: 35-47.

PAPIC C (2000) Regeneración de plántulas arbóreas sobre material leñoso en descomposición en un bosque sucesional de Chiloé, Chile. Tesis de Magíster en Ciencias, mención Biología, Facultad de Ciencias, Universidad de Chile, Santiago, Chile. 44pp.

PICKETT STA, SL COLLINS \& JJ ARMESTO (1987) Models, mechanisms and pathways of succession. The Botanical Review 53: 335-371.

SOKAL RR \& FJ ROHLF (1995) Biometry: the principles and practice of statistics in biological research. Third edition. W.H. Freeman and Company, New York, New York, USA. 887 pp.

VAN BREEMEN N (1995) How Sphagnum bogs down other plants. Trends in Ecology and Evolution 10: 270-275.

VEIT H \& K GARLEFF (1996) Evolución del paisaje cuaternario y los suelos en Chile central-sur. En: Armesto JJ, C Villagrán \& MTK Arroyo (eds) Ecología de los bosques nativos de Chile: 29-50. Editorial Universitaria, Santiago, Chile.

VILLAGRÁN C, JJ ARMESTO \& R LEIVA (1986) Recolonización postglacial de Chiloé insular: evidencias basadas en la distribución geográfica y los modos de dispersión de la flora. Revista Chilena de Historia Natural 59: 19-39.

VILLAGRÁN C (1990a) Glacial climates and theirs effects on the history of vegetation of Chile. A synthesis based on palynologycal evidence from Isla de Chiloé. Review of Palobotany and Palynology 65: 17-24.

VILLAGRÁN C (1990b) Glacial, late glacial and post glacial climate and vegetation of the Isla Grande de Chiloé, southern Chile $\left(41-44^{\circ} \mathrm{S}\right)$. Quaternary of South America and Antarctic Peninsula 8: 1-15.

Editor Asociado: Julio Gutiérrez

Recibido el 25 de abril de 2006; aceptado el 25 de septiembre de 2006 